\title{
The relationship of eating behaviors, hunger control, and physical activity to weight loss and quality of life in laparoscopic adjustable gastric banding patients
}

\begin{abstract}
This retrospective, descriptive study explored the relationship of eating behaviors, hunger control, and physical activity to weight loss and quality of life in 134 patients who had laparoscopic adjustable gastric banding (LAGB) surgery. Based on the findings of this study, it is recommended that gastric banding patients participate in at least 150 minutes per week of moderate to vigorous physical activity. Additionally, clinicians should consider development of band adjustment protocols that achieve early reduction of hunger in the banding patient
\end{abstract}

Volume 3 Issue 5 - 2015

\author{
Nancy Baugh,' Nancy L McCain, ${ }^{2}$ AbouEl \\ Makarim Aboueissa,' Carolyn Driscoll,' \\ Ronald K. Evans,' Jo Lynne Robins' \\ 'University of Southern Maine, USA \\ ${ }^{2}$ Virginia Commonwealth University School of Nursing, USA
}

Correspondence: Nancy Baugh, Faculty, University of Southern Maine, 96 Falmouth Street, Portland, Maine 04 I04,Tel 8042183759/2077804064,Email nbaugh@usm.maine.edu

Received: March 14,20I5 | Published: December 04, 2015

Abbreviations: NIH, national institute of health; LAGB, laparoscopic adjustable gastric banding; EWL, excess weight loss; QOL, quality of life; CDC, centers of disease control; USDA, united states department of agriculture; IPAQ-Short, international physical activity questionnaire short form; MET, metabolic equivalent of each task

\section{Introduction}

The prevalence of morbid obesity is increasing at an alarming rate worldwide and is becoming a major public health problem in the United States. Because of the failure of conventional weight loss therapies for morbid obesity, the National Heart, Lung, and Blood Institute of the National Institutes of Health (NIH) recommends surgical therapy as the only intervention associated with long-term success. ${ }^{1}$ Laparoscopic adjustable gastric banding (LAGB) has been steadily increasing in popularity since approval by the FDA in 2001. Successful weight loss with the LAGB is defined as greater than 50percent of excess weight loss (EWL). Early studies on the effectiveness of the LAGB were encouraging, with most studies reporting the average percentage of EWL over 50percent and a low incidence of complications. A systematic review of 4594 studies published worldwide involving 28,980 gastric banding patients found an average EWL of 56percent with the most significant weight loss occurring in patients with a BMI of $>40 \mathrm{~kg} / \mathrm{mm}^{2}{ }^{2}$ A recently published retrospective analysis of 2,090 banding patients found a mean EWL of 52.9percent at 5years postoperatively. However, a review of individual studies show more inconsistent outcomes, with percentages of EWL ranging from less than 30percent to as much as 70 percent. $^{3}$ Two recently published studies found the majority of patients failed to achieve the benchmark of 50percent of EWL at 2 and 3years postoperatively. ${ }^{4}$

A number of studies have reported improved quality of life (QOL) after gastric banding surgery. ${ }^{3,5-8}$ A study by Freys et al., ${ }^{9}$ reported a significant improvement in QOL in 73 banding patients but reported a high number of complications (38\%) that resulted in reoperation. A more recent study by Clough et al. ${ }^{10}$ reported marked improvement in physical, mental, emotional and social QOL in gastric banding patients over the age of 60 although they experienced less excess weight loss than their younger cohorts.

The primary weight loss mechanism of the gastric band is to produce early and prolonged satiety ultimately leading to reduced intake and weight loss. Appropriate fluid volume in the gastric band is a crucial factor in maintaining an increased sense of satiety and reduced hunger. Although the exact mechanism for this physiologic response is unclear, it is postulated to be related to physical restriction at the gastro-esophageal junction resulting in delayed gastric emptying. There are no studies on the relationship between hunger control and weight loss and very few guidelines in the literature to assist the bariatric team in achieving early satiety in this patient population.

The strong inverse relationship between physical activity and obesity has been well documented. ${ }^{11}$ The American Dietetic Association maintains that successful weight management requires not only a healthy diet but also daily physical activity. ${ }^{12}$ The Centers of Disease Control (CDC) and the United States Department of Agriculture (USDA) also recognize physical activity for its contribution to energy expenditure and weight maintenance. ${ }^{13-15}$ These organizations vary in their specific recommendations for physical activity for weight management, ranging from 150 minutes of moderate aerobic exercise per week (USDA) to 60 to 90minutes of moderate exercise on most days of the week (CDC) ${ }^{14,15}$ Although there are relatively few studies on the role of physical activity on weight loss outcomes after obesity surgery, several recent studies suggest that participation in regular activity after gastric bypass surgery results in better weight loss and maintenance. ${ }^{16-18}$ There are even fewer studies on the relationship between physical activity and weight loss in gastric banding patients. In one study, Colles et al., ${ }^{19}$ found regular walking was associated with better weight outcomes after one year in gastric banding patients. Chevallier et al., ${ }^{20}$ conducted a nationwide study in France to determine what factors predicted success of weight loss in banding patients. Factors associated with success in that study included change in eating habits and regular participation in physical activity. Those who exercised lost 2.3times more weight than those who did not. A 
recent study by Thalheimer et al., ${ }^{21}$ found sedentary behavior to be a significant $(p=0.045)$ predictor of failure to lose weight in 85 gastric banding patients. A better understanding of the relationship between physical activity and weight loss after gastric banding surgery has implications for planning treatment strategies to improve weight loss for each patient.

Patients with pre-surgical eating disturbances such as grazing, sweets eating, and binging may have suboptimal weight loss after gastric banding. Excessive consumption of sweetened beverages such as sodas and sweet tea is common in morbidly obese patients and has been associated with inferior weight outcomes after restrictive weight loss surgeries..$^{22}$ Himpens et al., ${ }^{23}$ found sweets eating behaviors to persist and to be a cause of failure after restrictive surgeries such as gastric banding. Sugarman et al., ${ }^{24}$ proposed that after restrictive surgeries, sweets eaters do not lose as much weight as non-sweets eaters; however, Dixon \& O'Brien ${ }^{25}$ found no difference in their banding patients. This finding was supported in a study by Hudson, Dixon et al., ${ }^{26}$ who found that a preference for sweets did not result in inferior weight loss in banding patients.

Colles et al., ${ }^{19}$ found that uncontrolled eating after gastric banding was associated with poor weight loss. However, a recent systematic review suggests that although uncontrolled eating behaviors may persist in some patients after surgery, in the majority of banding patients, overeating behaviors were reduced. It is speculated that reduction in overeating is related to the restrictive nature of the band. ${ }^{27}$

This article reports the findings of a study exploring the association of eating behaviors, hunger control and physical activity with weight loss and quality of life in LAGB patients. The results of this study will assist clinicians caring for this patient population in developing effective treatment strategies to optimize successful weight loss and maintenance.

\section{Methods}

\section{Design/aims}

The primary aim of this retrospective, descriptive study was to examine the impact of eating behaviors, hunger control and physical activity on weight loss and quality of life in LAGB patients. The independent variables were physical activity, eating behaviors and hunger control. The dependent variables were percentage of excess weight loss and quality of life at one and two years postoperatively. The secondary aim of the study was to determine whether successful weight loss at one year predicted successful weight loss at two years.

\section{Sample}

Participants in the study were 134 patients aged 15-70years old who underwent LAGB at a university healthcare system between December 2004 and December 2008. To be eligible for surgery patients required a BMI $>40 \mathrm{~kg} / \mathrm{m}^{2}$, or greater than $35 \mathrm{~kg} / \mathrm{m}^{2}$ with associated comorbidity and failed previous, significant attempts to lose weight.

\section{Procedures}

Institutional review board approval was obtained from the university to conduct a retrospective study on patients who received gastric banding from 2004 to 2008. At the initial consultation, data regarding demographics, weight, BMI, EWL, comorbidities and eating behaviors were collected. Patients completed study questionnaires about quality of life and physical activity at the initial visit and at each postoperative visit. Patients rated their level of hunger and satiety at each postoperative visit using a Likert type scale.

\section{Measures}

Anthropometric: percent age of excess weight loss is the preferred means of reporting weight loss in the morbidly obese after bariatric surgery and is based on the patients' ideal body weight. percentage of excess weight loss is calculated as (weight loss)/(excess weight) $\mathrm{X}$ 100 , with excess weight defined as weight above ideal body weight.

Physical activity: The International Physical Activity Questionnaire short form (IPAQ-short) has been used extensively to measure health related physical activity in adults. The form is a self-report measure that assesses physical activity undertaken across a comprehensive set of domains. Questions are asked regarding the duration and intensity spent in physical activities at work, as part of house and yard work, and in spare time for recreation, exercise and sports in the previous 7 days. The metabolic equivalent of each task (MET) can be calculated to provide information about the volume of activity. The subjects are categorized as "sufficiently active" or "insufficiently active" to maintain health. "Sufficiently active" is defined as

a. vigorous activity 3 or more days per week for at least 20 minutes per day, or

b. Moderate activity and/or walking 5 or more days per week for at least 30 minutes a day or,

c. Any activity on 5 or more days per week with a total of 600 or more METS/minutes per week.

Participation in health enhancing physical activity ("HEPA active") is defined as

a. Aigorous activity on 3 or more days a week with a total of 1500 vigorous METS/minutes per week, or

b. Any activity on 5 or more days per week totaling 3000 or more METS/minutes per week. The HEPA score exceeds the current $\mathrm{CDC}$ and FDA public health recommendations for physical activity.

The IPAQ short form has demonstrated good reliability and acceptable validity properties when compared with other established self-reports of physical activity. Craig et al., ${ }^{28}$ found the tool correlated well with pedometer readings $(r=0.30)$. A recent study by Papanthanasiou et al. ${ }^{29}$ found acceptable correlations $(r=0.37-0.47)$ between the IPAQ and exercise capacity on a treadmill.

Quality of life: The SF-36is one of the most widely used tools for self-reported health-related quality of life. It is a generic health survey geared to adults age 18years and older that consists of 36 questions yielding eight domains of functional health and well-being. The physical functioning, role limitations and vitality dimensions assess the extent to which the respondents perceive that their quality of life is influenced by their physical condition. The extent to which bodily pain hinders their ability to perform activities of daily living is also assessed. The mental component includes social functioning, role limitations due to emotional problems and general mental health. A Likert-type scale of rating produces domain raw scores, which are transformed into a 0 to 100 scale, with higher numbers reflecting higher functioning and quality. The scores are calibrated so that 50 is the average score or norm with a standard deviation of 10 . The SF-36 has demonstrated sound psychometric properties in a number of studies..$^{30,31}$ Ware et al., ${ }^{32}$ found the tool to have high internal 
consistency and test-retest reliability. Fallide \& Ramos ${ }^{33}$ found the SF-36 to have high internal consistency, reporting Cronbach's alphas of 0.72 to 0.94 )

Eating behaviors: Information about eating behaviors was obtained at the initial consultation by the dieticians on the bariatric team. Using food diaries and patient self-report the dieticians determined whether patients engaged in the following behaviors: sweets eating, drinking sugar sweetened beverages, eating junk food, volume eating, alcohol use and history of other eating disorders defined as follows:

Sweets eater: Someone who consumes over 300calories of sweet foods (e.g., candy, cookies, cakes, pies, ice cream, etc.) at least 3 times per week.

Sweetened beverage drinker: Someone who routinely chooses to drink regular sweetened beverages (e.g., sodas, tea, Kool-aid) rather than dietetic versions.

Junk food eater: Someone who routinely consumes over 300calories of non sweet junk foods (e.g. chips, pretzels, high fat microwave popcorn, pre-packages cheese crackers, etc.) at least 3times a week.

Volume eater: Someone who reports they consume larger than normal portions or who goes back for seconds on a routine basis.

Alcohol overuse: Alcohol Overuse is defined as reported intake of greater than one standard alcoholic beverage per day for females and greater than 2 standard alcoholic drinks per day for males.

Hunger: At each follow-up visit the patients were assessed by the bariatric team for their level of hunger using a 4-point frequency scale: $0=$ none, $1=$ rarely, $2=$ occasionally, $3=$ frequently.

\section{Data analysis}

Data were entered into an Excel spreadsheet and imported into the Statistical Package for the Social Sciences $\left(17^{\text {th }}\right.$ version $)$ for analysis. Descriptive statistics were used to analyze demographics. Chi square tests of independence were used to examine the relationship between physical activity behaviors, eating behaviors, hunger scores and percentage of EWL. For each chi square analysis, patients were grouped by weight loss categories of less than or equal to $24.9 \%$, $25-49-9 \%$ and greater than or equal to 50percent EWL. One-way analysis of variance was performed to assess the change in health related quality of life in LAGB patients from preoperatively to two years post-operatively. Linear regression was performed to determine if successful weight loss at one year predicted successful weight loss at two years.

\section{Results}

A demographic profile of the study participants is presented in Table 1 . Most of the participants were female (84\%). Caucasians accounted for the majority (59\%) of the group; African Americans comprised 38percent, Hispanics 2percent, and 1percent was classified as "other."

\section{Weight Loss}

Weight loss outcomes for years one and two are summarized in Table $2 \& 3$. The mean EWL for both years is less than 50percent. The mean preoperative BMI was $44.1 \pm 5.1$; the mean BMI at year one was $36.7 \pm 5.1$ and the mean BMI for year two was $35.9 \pm 6.7$. At year one, 30 patients $(23 \%)$ achieved a weight loss of $>50$ percent EWL; 40
(29\%) patients achieved a weight loss of between 25 and 49.9percent EWL; and the remaining 64 patients (48\%) achieved an excess weight loss of less than 25 percent. Of the 64 patients with data at 2 years postoperatively, $23(34 \%)$ experienced a greater than 50percent EWL; 20 patients $(32 \%)$ achieved a weight loss of between 25 and 49.9percent excess weight loss; and the remaining 21 patients (34\%) achieved less than 25 percent EWL at 2 years. Interestingly, 23 patients gained weight between years one and two. Of those gaining weight, $12(52 \%)$ had lost less than 25percent EWL in year one, 7 patients (30\%) had lost between 25 and $49.9 \%$ EWL and 4 (17\%) had lost greater than 50percent EWL in year one.

Table I Demographic characteristics of sample $(N=\mid 34)$

\begin{tabular}{ll}
\hline & Mean $=46.56$ years \\
Age & $\begin{array}{l}\text { Range }=15-70 \text { years } \\
\text { Median }=42 \text { years }\end{array}$ \\
\hline Gender & $1 \mathrm{I} 3(84 \%)$ \\
\hline Female & $21(16 \%)$ \\
\hline Ethnicity & $79(59 \%)$ \\
\hline Caucasian & $5 \mathrm{I}(38 \%)$ \\
African American & $3(2 \%)$ \\
Hispanic & $\mathrm{I}(<1 \%)$ \\
Other & $44.1 \pm 5 . \mathrm{I}$ \\
BMI kg/m & $272.6 \pm 4 \mathrm{I}$ \\
Weight (lbs) &
\end{tabular}

Table 2 Weight loss at one year $(N=134)$

\begin{tabular}{llllll}
\hline & Mean & Median & SD & Minimum & Maximum \\
\hline Weight & 226.5 & 224.8 & 37.7 & 151 & 345 \\
BMI & 36.7 & 36.2 & 5.1 & 24.8 & 48.7 \\
Pounds lost & 46.1 & 39.8 & 24.4 & 2.4 & 135 \\
\% EWL & 34.6 & 31.8 & 16.7 & 1.9 & 82.2
\end{tabular}

Table 3 Weight loss at two years $(N=64)$

\begin{tabular}{llllll}
\hline & Mean & Median & SD & Minimum & Maximum \\
\hline Weight & 223 & 219.7 & 46.8 & 121.7 & 334 \\
BMI & 35.9 & 35.8 & 6.7 & 20.3 & 50.9 \\
Pounds lost & 51.3 & 47 & 30.4 & -8 & 120 \\
\% EWL & 39.7 & 38.6 & 25 & -8.5 & 114.1 \\
\hline
\end{tabular}

\section{Quality of Life}

As shown in Figure 1 mean scores for the domains of physical functioning, physical role limitations, general health perceptions, vitality, social functioning, and overall physical health improved significantly between preoperatively and year one $(p<0.05)$. Additionally, the mean scores for the domains of physical functioning, physical role limitations, general health perceptions, vitality, social functioning, and overall physical health improved significantly 
between preoperatively and year two $(p<0.05)$. Reports of bodily pain decreased significantly from preoperatively to year one and year two $(p<0.05)$. There was no significant improvement in emotional functioning or overall mental health from preoperatively to year one or year two.

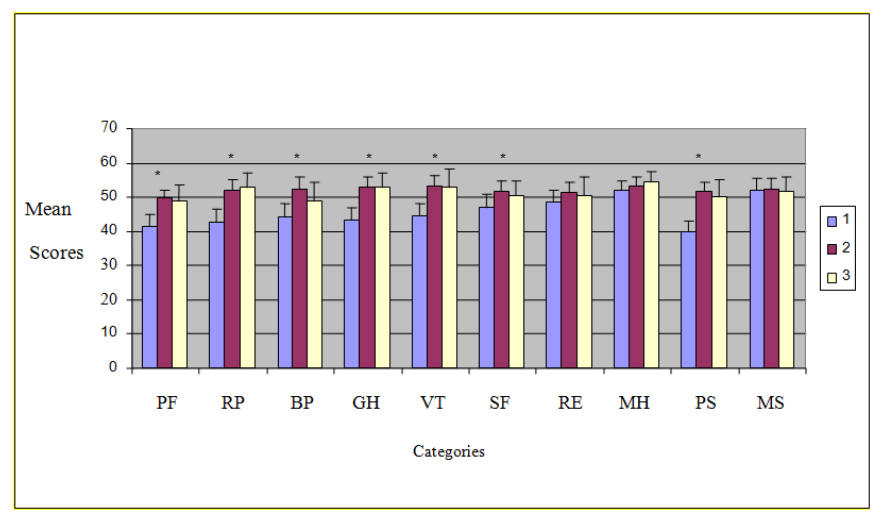

Figure I Quality of life scores in gastric banding patients.

\section{Physical activity}

The average time spent sitting per day, walking per week and participating in total physical activity per week preoperatively and at one and two years was calculated. The average minutes spent sitting per day was determined to be 432 minutes preoperatively (range $=210-1180 \mathrm{~min} /$ day). This number decreased to an average of 324 minutes per day at one year (range $=0-900 \mathrm{~min} /$ day) but increased to 363 minutes per day at two years (range $=30-1080 \mathrm{~min} /$ day). At year one, there was no association between the minutes spent sitting per day and the percentage of EWL. There were insufficient data to analyze the association between sitting and weight loss at year two.

Preoperatively, the patients in this sample walked an average of 178 minutes per week (range $=0-1680 \mathrm{~min} /$ week). The average time spent walking per week increased to $387 \mathrm{~min} /$ week (range $0-1680 \mathrm{~min} /$ week) at one year but decreased to $307 \mathrm{~min} /$ week at two years (range $0-1680 \mathrm{~min} /$ week). There was no association between the number of minutes walked per week and the percentage of excess weight loss at years one or two.

The average total minutes of physical activity in METS/minutes was determined to be $1053 \mathrm{~min} /$ week preoperatively (range $=0$ -
$9,144 \mathrm{~min} /$ week). The average at one year increased to $3317 \mathrm{~min} /$ week at one year (range $=0-21,546 \mathrm{~min} /$ week) but decreased to $2686 \mathrm{~min} /$ week at two years (range $=0-14,746 \mathrm{~min} /$ week). There was no evidence to support an association between total minutes of physical activity in METS/minutes per week and percentage of EWL $\left(\chi^{2}=6.21, p=0.184\right)$. However, a significant association between total physical activity in METS /minutes and percentage EWL was found at year two $\left(\chi^{2}=\right.$ $16.263, p=0.003)$.

At year one, $33.3 \%$ of the patients were participating in health enhancing physical activity (HEPA) and by year two this had decreased to $28.3 \%$. There was a significant positive $\left(\chi^{2}=5.985\right.$, $p<0.05)$ relationship between HEPA participation and percentage of excess weight loss at one year, however there was no relationship at year two $\left({ }^{2}=1.948, p=0.378\right)$. At year one, $57.5 \%$ of patients could be described as participating in sufficient activity. Although sufficient activity increased to $71.4 \%$ by year two, there was no significant relationship between participation in sufficient activity and percentage of EWL at one year or two years.

By year one, 37.9percent of patients were participating in greater than 150 minutes of moderate or vigorous physical activity per week. There was a significant positive association between participation in greater than 150 minutes per week in moderate or vigorous activity and percentage of excess weight loss at one year. $\left(\chi^{2}=7.356, p=0.025\right)$. The percentage of patients participating in this activity increased to $44.8 \%$ by year however the association with EWL was not significant by year two.

\section{Eating Behaviors}

Table 4 shows the percentage of EWL at one and two years for each identified eating category: sweets eaters, sweetened beverage drinkers, junk food eaters, volume eaters and alcohol users. The relationship between sweets eating and drinking sweetened beverages and percentage of EWL was not statistically significant at year one or year two. There was not a significant relationship between eating junk food regularly and amount of weight loss at year one and year two. Additionally, there was not a significant relationship between being a volume eater and weight loss at year one and year two. Overall use of alcohol was low in this cohort at $20 \%$, which is less than the US average of $64 \%$ for adults. ${ }^{34}$ There was no relationship between regular alcohol use and the percentage of EWL at year one and year two.

Table 4 Eating behaviors and percentage of excess weight loss $(E W L)$ at one year $(N=134)$ and two years $(N=64)$

\begin{tabular}{llll}
\hline & $<24.9 \%$ EWL & $\mathbf{2 5 - 4 9 . 9 \%}$ EWL & $>$ 50\% EWL \\
\hline & I Year/ 2 Year & I Year/ 2 Year & I Year/ 2 Year \\
\hline Sweets eaters & $28 \% / 27 \%$ & $30 \% / 31 \%$ & $30 \% / 31 \%$ \\
Sweetened Beverage Eaters & $22 \% / 22 \%$ & $25 \% / 25 \%$ & $15 \% / 15 \%$ \\
Junk Food Eaters & $22 \% / 23 \%$ & $27 \% / 27 \%$ & $18 \% / 17 \%$ \\
Volume Eaters & $24 \% / 22 \%$ & $28 \% / 29 \%$ & $22 \% / 21 \%$ \\
Alcohol users & $5 \% / 5 \%$ & $8 \% / 9 \%$ & $7 \% / 6 \%$ \\
\hline
\end{tabular}

\section{Hunger and satiety}

At year one, $76 \%$ of patients who experienced adequate weight loss ( $>50 \%$ EWL) reported feeling occasionally hungry or not hungry at all for most of the day. For those patients losing less than $24 \%$
EWL, 67\% reported feeling constantly or often hungry throughout the day. There was a significant positive relationship between degree of hunger reported by patients and successful weight loss at one year $\left(\chi^{2}=27.92, p<0.05\right)$. At two years, $42 \%$ of patients with successful weight loss reported feeling occasionally or not hungry, whereas 
among those with less than $24 \%$ EWL, $52 \%$ were often or constantly hungry. There was not a significant relationship between degree of hunger and weight loss at year two.

\section{One-year weight loss as a predictor of two- year weight loss}

There was statistical evidence of a positive linear relationship between two-year and one-year weight loss. $\left(R^{2}=58.2 \%, p<0.05\right)$. Successful weight loss during the first year after gastric banding predicts weight loss in year two.

\section{Discussion}

Patients in this sample demonstrated high variability in weight loss outcomes after gastric banding surgery and the percentage of EWL was not as dramatic for this cohort as compared with literature reports of patients who had gastric banding at other institutions worldwide. Successful weight loss after a bariatric procedure is defined as greater than 50percent EWL. Weight loss outcomes for patients in this study were 34.6percent at one year and 39.7percent at two years. Long-term success of gastric banding is dependent on frequent follow up and aggressive adjustments of the band. However, there are few guidelines and very little data available in the literature to assist practitioners in developing a standardized approach to frequency of follow-up visits and adjustments of the band. Some of the differences in weight loss outcomes in this study may be attributed to the experience of the bariatric team. As the years unfolded, the team developed and then frequently revised patient care procedures based on patient experiences and feedback. After several years of "trial and error" the team agreed the patients should have their first "fill" at six weeks, and then return every month until they were no longer hungry (according to a self-rating scale) and were losing one to two pounds per week and not experiencing symptoms of obstruction. Generally, patients received one to two milliliters of fluid in their bands at the initial visit and then 0.5 to $1 \mathrm{ml}$ at subsequent visits until they were "optimally" filled.

Although weight loss outcomes were less than the established success rate of greater than 50percent EWL, most patients lost weight and demonstrated an improvement in health related quality of life. Patients reported significant improvement in their ability to perform physical activity in general (physical functioning and physical summary scores), accomplish tasks (role physical), and participate in social activities (social functioning). Additionally, patients reported significantly less bodily pain and increased energy levels (vitality). They reported only minimal improvements in emotional and mental health. Improvement in quality of life may have been influenced by many factors such as reinforcement by the bariatric team at follow-up visits and participation in support group meetings.

Physical activity data were collected at each visit using the IPAQ questionnaire which depends on self-report of frequency, duration and intensity in participation of physical activities. Patients in this cohort decreased the amount of sitting from preoperatively to year one but increased by year two. Furthermore, no relationship was found between amount of minutes sitting per day and weight loss. Patients increased participation in minutes of walking per week from preoperatively to year one but decreased walking by year two. There was no relationship found between the minutes of walking per week and percentage of EWL. The total physical activity in METS/minutes increased from preoperatively to year one and although this activity decreased by $631 \mathrm{METS} /$ minutes per week by year two, there was a significant positive relationship in engagement in this behavior with percentage of EWL by year two $(p=0.003)$.
Although participation in physical activities overall decreased from year one to year two, there was a significant positive relationship between participation in greater than 150 minutes per week of moderate or vigorous physical activity and adequate weight loss $(p=0.025)$ and participation in health enhancing physical activities and adequate weight loss $(p<0.05)$. These results support recommendations of previous studies (Evans et al, 2007; Evans, 2010) for at least 150 minutes per week of moderate to vigorous physical activity to maintain weight loss after bariatric surgery. Factors that contribute to a decrease in physical activities at year two should be explored and interventions developed to assist patients in enhancing and maintaining participation in sufficient physical activity.

Patients in this study were classified as sweets eaters, sweetened beverage drinkers, regular junk food eaters, volume eaters or regular users of alcohol using criteria developed by the bariatric team. There was no statistically significant relationship noted between any of these eating behaviors and the percentage of EWL at one or two years. Patient numbers in each eating category were fairly well distributed among each weight loss category. For example, although it was anticipated that a higher percentage of patients who lost less than 24percent of EWL would be sweets eaters, sweetened beverage drinkers, junk food eaters and volume eaters, this was not the case. In fact, there were higher percentages of sweets eaters, sweetened beverage drinkers and junk food eaters among those who lost between 25 and $49.9 \%$ EWL.

It had been assumed by the bariatric team that a reduction in hunger was partially responsible for weight loss in gastric banding patients. This assumption was supported by the findings of this study in that there was a significant inverse relationship between patient reports of decreased hunger and successful weight loss at year one $(p<0.05)$. Based on the findings of this study, clinicians should consider development of adjustment protocols that achieve early reduction of hunger in the banding patient.

Although $23(36 \%)$ patients gained weight by year two, there was a significant positive relationship between weight loss between years one and two $(p<0.05)$. The relationship indicated that successful weight loss at one year predicted continued weight loss at two years. The majority of patients lost an average of $5.1 \%$ more weight between years one and two.

\section{Conclusion}

Limitations of this study included the subjective nature of questionnaires used for data collection and the results were limited to one and two year data. Objective measures of physical activity such as pedometers should be considered for future studies. Longitudinal studies are needed that focus on behavioral and environmental influences on weight outcomes in gastric banding patients.

\section{Acknowledgements}

None.

\section{Conflict of interest}

The author declares no conflict of interest.

\section{References}

1. NIH National Institutes for Health. Clinical Guidelines on the Identification, Evaluation, and Treatment of Overweight and Obesity in Adults-The Evidence Report. National Institutes of Health, National Heart, Lung, and Blood Institute; 1998. 
2. Cunneen SA. Review of meta-analysis comparisons of bariatric surgery with a focus on laparoscopic adjustable gastric banding. Surg Obes Relat Dis. 2008;4(Suppl 3):S47-S55

3. Ahron SH, Montgomery KF, Watkins BM. Laparoscopic adjustable gastric banding: Weight loss, comorbidities, medications usage and quality of life at one year. Obes Surg. 2005;15(5):641-647.

4. Kasza J, Brody F, Vaziri K, et al. Analysis of poor outcomes after laparoscopic adjustable gastric banding. Surgical Endoscopy. 2011;25(1):41-47.

5. Weiner R, Datz M, Wagner D, et al. Quality of life outcome after laparoscopic gastric banding for morbid obesity. Obes Surg. 1999;9(6):539-545.

6. Schok M, Grenen R, van Antwerpen T, et al. Quality of life after laparoscopic adjustable gastric banding surgery: Postoperative and retrospective preoperative evaluations. Obes Surg. 2000;10(6):502-508.

7. Dixon AF, Dixon JB, O'Brien PE. Laparoscopic adjustable gastric banding induces prolonged satiety: A randomized blind crossover study. J Clin Endocrinol Metab. 2005;90(2):813-819.

8. Horchner R, Tuinebreijer MW, Kelder PH. Quality of life assessment of morbidly obese patients who have undergone a Lap Band operation:2 year follow-up study. Is the SF-36 a useful instrument to measure quality of life in morbidly obese patients? Obes Surg. 2001;11(2):212-218.

9. Frey SM, Tigges A, Heimbucher J, et al. A Quality of life following laparoscopic gastric banding in patients with morbid obesity. Journal of Gastrointestinal Surgery. 2001;5(4):401-407.

10. Clough A, Layin L, Shah A, et al. Laparoscopic gastric banding in over 60's. Obes Surg. 2011;21(1):10-17.

11. King GA, Fitzhugh EC, Bassett DR, et al. Relationship of leisure time physical activity and occupational activity to the prevalence of obesity. International Journal of Obesity and Related Metabolic Disorders. 2001;25(5):606-612.

12. Seagle HM, Strain GW, Makris A, et al. Position of the American Dietetic Association: Weight management. J Am Diet Assoc. 2009;102(2):330346.

13. USDA exercise guidelines. 2005 .

14. CDC-Centers for Disease Control. Obesity Trends by US State 1985 2010.

15. Poirier P, Despree JP. Exercise in weight management of obesity. Cardiol Clin. 2001;19(3):459-470

16. Evans RK, Bond DS, Wolfe LG, et al. Participation in $150 \mathrm{~min} / \mathrm{wk}$ of moderate or higher intensity physical activity yields greater weight loss after gastric bypass surgery. Surg Obes Relat Dis. 2007;3(5):526-530.

17. Bond D, Leahey TM, Vithiananthan S, et al. Bariatric surgery for severe obesity: The role of patient behavior. Med Health R I. 2009;92(2):59-60.

18. Hatoum IJ, Stein HK, Merrifield BF, et al. Capacity for physical activity predicts weight loss after Roux en Y gastric bypass. Obesity. 2008;17(1):92-99.
19. Colles SL, Dixon JB, O’Brien PE. Hunger control and regular physical activity after laparoscopic adjustable gastric banding. Obes Surg. 2008;18(7):833-840.

20. Chevallier JM, Paita M, Rodde-Dunet MH, et al. Predictive factors of outcome after gastric banding:A nationwide survey of the role of center activity and patient's behavior. Ann Surg. 2007;246(6):1034-1039.

21. Thalheimer A, Bueter M, Wierleman A, et al. Predictability of outcome in laparoscopic gastric banding. Obes Facts. 2009;2(Suppl 1):S27-S30.

22. Busetto L, Valente $\mathrm{P}$, Pisent $\mathrm{C}$, et al. Eating patterns in the first year following laparoscopic adjustable silicone gastric banding for morbid obesity. Int J Obes Relat Metab Disord. 1996;20(6):539-546.

23. Himpens J, Dapri G, Cadiere. A prospective randomized study between laparoscopic gastric banding and laparoscopic isolated sleeve gastrectomy: Results after 1 and 3years. Obes Surg. 2006;16(11):1450 1456.

24. Sugerman HJ, Kellum JM, Engle KM, et al. Gastric bypass for treating severe obesity. Am J Clin Nutr. 1992;155(2 Suppl):S560-566.

25. Dixon JB, O'Brien PE. Changes in comorbidities and improvement in quality of life after LAP-band placement. Am J Surg. 2002;184(6B):51S$54 \mathrm{~S}$.

26. Hudson S, Dixon JB, O'Brien PE. Sweets eating is not a predictor of outcome after lap band placement: Can we finally bury the myth? Obes Surg. 2002;12(6):789-794.

27. Dodsworth A, Warren-Forward H, Baines S. Changes in eating behavior after laparoscopic adjustable gastric banding: A systematic review of the literature. Obes Surg. 2010;20(11):1579-1593.

28. Craig CL, Marshall AL, Sjostrom M, et al. International physical activity questionnaire:12-country reliability and validity. Med Sci Sports Exerc. 2003;35(8):1381-1395

29. Papanthaasiou G, Georgoudis G, Georgakopoulos C, et al. Criterion related validity of the short International Physical Activity Questionnaire against exercise capacity in young adults. Eur J Cardiovasc Prev Rehabil. 2010;17(4):380-386.

30. Elliott TE, Renier CM, Palcher JA. Chronic pain, depression, and quality of life: Correlations and predictive value of the SF-36. Pain Med. 2003;4(4):331-339.

31. McHorney CA, Ware JE, Rogers W, et al. The validity and relative precision of MOS Short and Long Form Health Status Scales and Dartmouth COOP Charts. Med Care. 1992;30(5 Suppl):MS253-MS265.

32. John E Ware. A New Chapter for the SF-36v2 $2^{\mathrm{TM}}$ Health Survey. 2nd ed Quality Metric Incorporated, 2007.

33. Fallide I, Ramos I. Validity and reliability of the SF-36 Health Survey questionnaire in patients with coronary artery disease. $J$ Clin Epidemiol. 2000;53(4):359-365.

34. Lydia Saad. Drinking Habits Steady Amid Recession. Gallup. 2009 